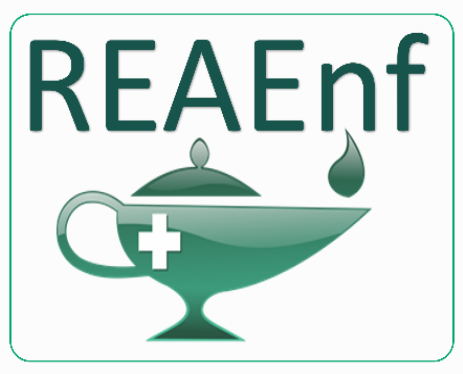

Revista Eletrônica Acervo Enfermagem
REVISÃO BIBLIOGRÁFICA

Recebido em: 10/2020

Aceito em: 11/2020

Publicado em: 12/2020

\title{
Investigação bibliográfica sobre medidas preventivas da infecção do trato urinário
}

\author{
Bibliographic investigation on preventive measures for urinary tract infection
}

Investigación bibliográfica sobre medidas preventivas para infección del tracto urinario

Fábio Manoel Gomes da Silva1*, Dhyellen Daynara Sales Sacramento².

\begin{abstract}
Resumo: A Infecção Relacionada à Assistência à Saúde (IRAS) é um grave problema de saúde pública no Brasil, sendo responsável pelo aumento dos casos de morbimortalidade, bem como pelos longos períodos de internação hospitalar. Este artigo tem como objetivo, através de uma Revisão de Literatura, apresentar acerca da abordagem epidemiológica e medidas preventivas das infecções do trato urinário relacionadas a cateterismo vesical. A discussão mostrou que a grande maioria das infecções hospitalares é causada por um desequilíbrio da relação existente entre a microbiota humana normal e os mecanismos de defesa do hospedeiro. Em síntese, é notório o importante papel da equipe de enfermagem na prevenção de infecção do trato urinário, pois é ela quem instala, mantém e retira a sonda vesical de demora, bem como de todos os profissionais da área da saúde, no que tange a adoção de medidas preventivas e campanhas educativas voltadas para o uso racional da sonda vesical, a fim de evitar as ITU por cateterismo de demora.
\end{abstract}

Palavras-chave: Investigação cientifica, Medidas de prevenção, Infecção urinária.

\begin{abstract}
Health Care Related Infection (HAI) is a serious public health problem in Brazil, responsible for the increase in cases of morbidity and mortality, as well as for long periods of hospitalization. This article aims, through a Literature Review, to present about the epidemiological approach and preventive measures of urinary tract infections related to bladder catheterization. The discussion showed that the vast majority of nosocomial infections are caused by an imbalance in the relationship between the normal human microbiota and the host's defense mechanisms. In summary, the important role of the nursing team in preventing urinary tract infection is notorious, as it is they who install, maintain and remove the delayed bladder catheter, as well as all health professionals, with respect to adoption of preventive measures and educational campaigns aimed at the rational use of the bladder catheter, in order to avoid UTI by delayed catheterization.
\end{abstract}

Keywords: Scientific investigation, Prevention measures, Urinary infection.

Resumen: Las Infecciones Relacionadas con la Atención de la Salud (IAAS) son un grave problema de salud pública en Brasil, responsable del aumento de casos de morbilidad y mortalidad, así como de largos períodos de hospitalización. Este artículo tiene como objetivo, a través de una Revisión de la Literatura,

1Universidad Europea del Atlântico, Belém - PA. *E-mail: enfermeirofabiogomez@gmail.com

${ }^{2}$ Secretaria De Saúde do Estado do Pará, Belém - PA.

REAEnf/EJNC | Vol. 6 | e5714 | DOI: https://doi.org/10.25248/REAenf.e5714.2020 Página 1 de 6 
presentar sobre el abordaje epidemiológico y las medidas preventivas de las infecciones del tracto urinario relacionadas con el sondaje vesical. La discusión mostró que la gran mayoría de las infecciones nosocomiales son causadas por un desequilibrio en la relación entre la microbiota humana normal y los mecanismos de defensa del huésped. En resumen, es notorio el importante papel del equipo de enfermería en la prevención de la infección del tracto urinario, ya que son ellos quienes instalan, mantienen y retiran la sonda vesical retardada, así como todos los profesionales de la salud, respecto a adopción de medidas preventivas y campañas educativas encaminadas al uso racional de la sonda vesical, con el fin de evitar la IU por sondaje tardío.

Palabras clave: Investigación científica, Medidas de prevención, Infección urinaria.

\section{INTRODUÇÃO}

A Infecção Relacionada à Assistência à Saúde (IRAS) é considerada um grande problema de saúde pública, sendo responsável pelo aumento da morbimortalidade dos pacientes, bem como do período de internação e custos assistenciais elevados. Dentre as IRAS, a Infecção do Trato Urinário (ITU) é considerada a mais comum, tendo a presença de cateter urinário de demora como o principal fator de risco, neste interim também pode contribuir consideravelmente para casos de insuficiência renal aguda ou crônica, dependendo da gravida e do quadro clínico apresentado pelo paciente, levando muitos usuários para o Serviço de Terapia Rena Substitutiva (ESTELA AE e OLIVEIRA AF, 2020).

A infecção do trato urinário (ITU) é uma inflamação das vias urinárias com sintomatologia e presença de bactérias na urina. A ITU é responsável por 35 a $45 \%$ de todas as Infecções Relacionadas à Assistência à Saúde (IRAS) adquiridas. Sendo que, em grande maioria dos casos, cerca de $80 \%$, está relacionada com a cateterização vesical. Entre os pacientes hospitalizados em Unidade de Terapia Intensiva (UTI), 10\% deles são expostos a este procedimento e ficam susceptíveis a ITU (MARKS FO, et al., 2020).

O termo infecção é caracterizado como uma doença causada pelo crescimento de germes patogênicos no organismo. Desta forma, para que o processo infeccioso se instale é imprescindível que haja a penetração do agente infeccioso no corpo do hospedeiro susceptível e haja a proliferação, multiplicação dos microrganismos, culminando na apresentação da sintomatologia. As infecções podem acometer diversas localizações topográficas de um indivíduo, ou disseminar-se pela corrente sanguínea. Alguns agentes têm "favoritismo" por determinadas partes do corpo, assim a localização da infecção depende do tipo de microrganismo (SILVA MR, et al., 2020).

Os referidos autores acrescentam ainda que das principais infecções hospitalares endêmicas, a contaminação do TU é na maioria das vezes, a mais comum. Seguida da infecção da ferida cirúrgica, infecções respiratórias e as bacteremias primárias. Dentro desta perspectiva, entende-se por infecção das vias urinárias a presença e multiplicação de microrganismos no aparelho urinário, com possível invasão e reação das estruturas tubulares do aparelho urinário ou órgãos anexos. Do ponto de vista clínico, a infecção do trato urinário (ITU) pode ser dividida em dois grupos: ITU inferior, onde a presença de bactérias se limita à bexiga (cistite), e do trato superior (pielonefrite), que se define como aquela que afeta a pélvis e o parênquima renal (SAKAI AM, et al., 2020).

As infecções urinárias figuram entre as infecções nosocomiais de maior frequência em hospitais gerais de 5 a 10\% ocorrendo após cistoscopias ou procedimentos cirúrgicos com manipulação do trato urinário. Então, pode-se perceber que apesar de todo o avanço técnico-científico a infecção por cateterismo vesical de demora continua sendo a que mais ocorre nas instituições hospitalares, haja vista o que consta nas próprias estatísticas e pesquisas relacionadas ao assunto, fato este que gerou o interesse do presente estudo, a fim de contribuir com a pesquisa e ensino na enfermagem (VAZ BC, et al., 2020).

Neste sentido, esta revisão da literatura visa contribuir com a discussão do tema em busca de perceber quais as principais medidas preventivas que podem ser adotadas no cuidado ao paciente para evitar infecções do trato urinário. Tem-se como objetivos: Analisar o perfil clínico e epidemiológico dos pacientes 
que fazem uso de cateter vesical nas Unidades de Terapia Intensiva (UTI); Relacionar o uso do cateter vesical com o aparecimento de Infecção do Trato Urinário; Identificar a relação entre tempo de permanência do cateter de demora no paciente e as complicações adquiridas durante a internação.

\section{MÉTODOS}

Trata-se de uma revisão narrativa da literatura que foi construída através de uma minuciosa investigação em um recorte temporal entre 2019 a 2020, realizada análise e síntese de artigos publicados nas demais revistas eletrônicas, pesquisadas nas principais bases cientificas contendo conteúdos direcionados ao profissional enfermeiro: Literatura Latino-americana e do Caribe em Ciências da Saúde (LILACS), Scientific Electronic Library Online (SCIELO), Centro Latino Americano e do Caribe de Informação em Ciências da Saúde (BIREME), Revista Brasileira de Enfermagem, dentre outras. Foram incluídos na pesquisa artigos publicados em língua portuguesa de acordo com os descritores: Epidemiologia. Infecção Hospitalar. Medidas Preventivas. Infecção do Trato Urinário. Traçando de forma temporal e cronológica, com referências atualizadas uma visão holística dos aspectos epidemiológicos, e as causas principais de quando e como um paciente assistido por uma equipe de enfermagem adquire de forma implícita uma infecção do trato urinário.

\section{RESULTADOS}

Mensurar de forma cronológica o retrato clínico e epidemiológico que podem se destacar em uma investigação cientifica com a análise e síntese de diversos acervos inclusos na temática o qual foram utilizados para construção uma média de 19 artigos pertinentes acerca de pacientes que fazem uso de cateter vesical nas Unidades de Terapia Intensiva (UTI), tendo como fator fundamental as infecções nosocomiais pela má ou inadequada manipulação dos mesmos sem as medidas preventiva de higienização; Observar equivalências de situações com o uso do cateter vesical com o aparecimento de Infecção do Trato Urinário; Verificar como profissional a relação entre tempo de permanência do cateter de demora no paciente e as complicações adquiridas durante a internação, consideradas as mais comuns infecções bacterianas ou fúngicas, responsáveis por 80 em cada 1.000 consultas clínicas. Além disso, cerca de $15 \%$ dos óbitos por insuficiência renal resultam de lesão secundária à infecção crônica renal. Conforme as ITUs ocorram com a devida frequência em crianças até os seis anos de idade e em mulheres jovens, a prevalência dessas infecções se eleva com a idade, passando a representar doença de grande importância em adultos idosos. Em recém-nascidos, cerca de $75 \%$ das ITUs ocorrem em crianças do sexo masculino e se instalam por via hematogênica. Em crianças com mais de três meses de idade, cerca de $90 \%$ das ITUs se manifestam no sexo feminino e podem causar cicatrizes renais quando associadas a refluxo vesico ureteral. É importante ressaltar ainda que entre 1\% e 1,5\% das meninas em idade escolar apresentam bacteriúria assintomática, que não danifica os rins, mas que torna este grupo particularmente suscetível a ITUs na idade adulta (COMIN D, et al., 2020).

Em mulheres adultas jovens existe um aumento da incidência de infecções urinárias sintomáticas, que parecem estar implicadas diretamente com o início da vida sexual. Paralelamente a isso, a incidência de bacteriúria assintomática aumenta com o passar dos anos, elevando-se de 1\% na infância para 15\% em mulheres adultas idosas. Nessas últimas, maior prevalência de ITUs parece depender de disfunções hormonais e neurológicas, que acarretam dificuldade de esvaziamento vesical. Em homens adultos jovens as ITUs são muito raras e, quando ocorrem, estão quase sempre implicadas com anomalias urinárias estruturais. Por outro lado, após os 60 anos existe, neste grupo, um aumento gradual de incidência de infecções urinárias que chega a atingir entre $3 \%$ e $4 \%$ dos homens e que se relaciona com quadros de hiperplasia prostática, aumento da próstata (SOUZA SM, et al., 2020).

Aproximadamente dois terços das IRAS são de origem autógena, significando o desenvolvimento da infecção a partir da microbiota do paciente, que pode ter origem comunitária ou intra-hospitalar. Em ambas as situações, a colonização precede a infecção, sendo difícil determinar se o paciente trouxe 0 microrganismo da comunidade ou adquiriu de fonte exógena durante a internação. $\mathrm{O}$ autor ainda acrescenta

REAEnf/EJNC | Vol. 6 | e5714 | DOI: https://doi.org/10.25248/REAenf.e5714.2020 Página 3 de 6 
que a infecção hospitalar, o hospedeiro é o elo mais importante da cadeia epidemiológica, pois alberga os principais microrganismos que na maioria dos casos desencadeiam processos infecciosos. A patologia de base favorece a ocorrência das IRAS por afetar os mecanismos de defesa anti-infecciosa: grandes queimaduras; a desnutrição; deficiências imunológicas; bem como o uso de alguns medicamentos e os extremos de idade. Também favorecem o desenvolvimento das infecções os procedimentos invasivos terapêuticos ou para diagnósticos, podendo veicular agentes infecciosos no momento de sua realização ou durante a sua permanência (PEREIRA ML, et al., 2020).

\section{DISCUSSÃO}

Salienta se que algumas IRAS são evitáveis e outras não. Infecções preveníeis são aquelas em que se pode interferir na cadeia de transmissão dos microrganismos. A interrupção dessa cadeia pode ser realizada por meio de medidas reconhecidamente eficazes, a principal delas, como sendo a lavagem das mãos, o processamento dos artigos e superfícies, a utilização dos equipamentos de proteção individual, no caso do risco laboral e a observação das medidas de assepsia (SILVA GF, et al., 2020).

As ITUs são, em geral, causadas por bactérias gram-negativas aeróbicas presentes na flora intestinal. Nas infecções urinárias agudas sintomáticas existe nítida predominância de Escherichia coli, enquanto que nas infecções crônicas ou adquiridas em ambiente hospitalar ou relacionadas com anomalias estruturais do trato urinário existe uma incidência mais equitativa das diferentes enterobactérias, com aumento da prevalência de infecções causadas por Klebisiella sp., Proteus sp., Pseudomonas sp., Enterobacter sp., Enterococos e por gram-positivos, como Staphylococcus (SÉ ACS, et al., 2020).

Quadros de carbúnculo renal, ou seja, abscessos corticais causados por Staphylococcus eram relativamente comuns no passado e decorriam de envolvimento hematogênico do trato urinário por estas bactérias. Estes quadros são raros na atualidade e, quando surgem, quase sempre estão implicados com infecção acedente por E. coli (SILVA LR, et al., 2020).

Para diferentes diagnósticos e processos terapêuticos, por vezes é indispensável o uso de meios invasivos, que implicam no manuseio direto ou indiretos do trato urinário, por meio de sondagem, irrigação vesical ou procedimentos relacionados. Muito embora os usos de cateteres uretrais tenham trazido grandes benefícios para inúmeros pacientes, a sua prática trouxe, também, problemas e riscos potenciais relacionados ao seu uso no trato urinário (HOFFMANN LY, et al., 2020).

Os principais fatores de risco relacionados a esta técnica são: sexo feminino, idade, doença de base, antibioticoterapia, presença do cateter, cateterização prolongada, manipulação das vias urinárias, qualidade do cuidado do cateter, abertura do sistema. Na contaminação pela via periuretral há diferenças entre os sexos quanto à forma de colonização. Em pacientes do sexo feminino, em torno de $70 \%$ das ITUs ocorrem quando, a partir da colonização do períneo e uretra, as bactérias alcançam a bexiga, através do muco periuretral que engloba e envolve o cateter. Já nos pacientes do sexo masculino, somente em um terço dos que desenvolveram bacteriúria relacionada ao cateter foi possível demonstrar as colonizações uretral e retal prévia pelo mesmo microrganismo. No homem, aparentemente, a maior parte das ITUs são causadas por contaminação do cateter e/ou sistema coletor, através das mãos dos profissionais de saúde ou do próprio paciente (BARBALHO AMDS, et al., 2019).

Outro fator de risco significante para aquisição de ITUs relacionado ao sexo feminino, provavelmente devido a curta extensão da uretra feminina facilitando o acesso de microrganismos da microbiota perineal para a bexiga. Reportaram se que a prevalência de ITUs foi maior em pacientes do sexo feminino, tanto em infecções comunitárias como de origem hospitalar. Mencionam se ainda que a frequência de contaminação de pacientes em uma mesma unidade, pelo mesmo microrganismo, demonstra a importância das mãos como fontes diretamente responsáveis pelas infecções cruzadas e pelos altos índices de ITU, revelando a necessidade de uma boa higienização no atendimento a cada paciente (ALENCAR PA, et al., 2020).

Dentro desta perspectiva, a via intraluminal a bacteriúria pode ocorrer, do lúmen do cateter vesical a partir da bolsa coletora de urina, ou quando da desconexão (desaconselhável explicitamente) do sistema

REAEnf/EJNC | Vol. 6 | e5714 | DOI: https://doi.org/10.25248/REAenf.e5714.2020 Página 4 de 6 
fechado, em dois locais: no tubo de drenagem do saco coletor com a finalidade de esvaziar o recipiente; na junção do cateter vesical e tubo coletor: nessas condições as bactérias penetram nos locais citados, se multiplicam e, por via retrógrada (TAVARES JMM, et al., 2020).

É perceptível que o cateter tem um importante papel na patogênese da ITU, uma vez que este provoca a quebra dos mecanismos de defesa, distendendo a uretra e bloqueando os ductos das glândulas periuretral. Além disso, o balão de retenção não permite o esvaziamento completo da bexiga mantendo urina residual. Os microrganismos podem se aderir à superfície externa do cateter e migrar ao longo deste. Dentro desta afirmativa é interessante salientar os principais fatores que predispõem o surgimento de uma infecção urinária em pacientes submetidos ao cateterismo vesical de demora, são: técnica imprópria da lavagem das mãos; inserção do cateter urinário sem a execução da técnica e assepsia incorretamente; sonda vesical desconectada do coletor de urina; saída do coletor de urina tocando a superfície contaminada; urina na sonda vesical ou coletor de urina, sendo permitido reentrar na bexiga (refluxo); irrigações repetidas da sonda vesical com soluções; o uso indiscriminado de cateterismo vesical, sem que haja indicação necessária; a permanência aumentada da sonda vesical, além da necessidade do paciente; a dimensão do cateter maior do que a apropriada para o paciente lesa os tecidos e favorece a colonização; o uso de balonetes maiores que o ideal faz com que aumente a quantidade de urina residual, aumentando a probabilidade de infecções (BRÍGIDO HPC, et a., 2020).

Cateteres com cuffs maiores do que $10 \mathrm{ml}$ devem ser reservados para situações com indicação específica, como é o caso de algumas cirurgias ou em mulheres com rompimento da musculatura pélvica. Acrescentariam que os riscos de infecção relacionados aos procedimentos diagnósticos e terapêuticos podem ser pormenorizados, uma vez que dependem diretamente da capacitação de recursos humanos (PORTELA FM, et al., 2019).

Esses fatos parecem indicar a real necessidade de um investimento anterior à prática profissional, na formação acadêmica, por exemplo, que tem se mostrado deficiente quanto ao ensino e a prática acerca do controle de infecção. Acrescentam se ainda muitas formas de prevenção de ITU, relacionado ao cateterismo vesical de demora: retirada precoce do cateter; posicionamento adequado do sistema; técnica asséptica na passagem do cateter; educação continuada; ao transferir o paciente do leito, lembrar sempre de clampear o tubo do saco coletor para que não haja refluxo da urina da bolsa coletora para dentro da bexiga (MARTINS PCA e VAZ AKMG, 2020).

Outra forma de prevenir a infecção no trato urinário é a realização da técnica asséptica, ou seja, com a utilização da luva estéril, a higiene íntima prévia e a antissepsia, visto que, a ação dos antissépticos é rapidamente inativada pela presença de matéria orgânica. Atualmente, os estudos mostram que a sequência recomendada para antissepsia do cateterismo vesical, tem sido iniciada pelo meato uretral, uma vez que se estabelece a ordem de realização do procedimento pelo local menos contaminado para o de maior contaminação. A antissepsia é o processo de eliminação ou inibição do crescimento dos microrganismos na pele e mucosas. Ela é realizada com uso de antissépticos que são formulações hipoalérgicas. O preparo da região genital para o procedimento de cateterização vesical deve ser realizado com solução aquosa de iodóforos, deste modo corroborando com a redução de riscos inerentes de possíveis contaminações no ato do procedimento realizado por profissionais como: Enfermeiros e Médicos (FERRACINI TS e RYMSZA T, 2020).

\section{CONSIDERAÇÕES FINAIS}

De acordo com a metodologia empregada e os resultados obtidos no presente estudo, sobre as abordagens epidemiológicas e medidas preventivas acerca das ITU, em unidades de terapia intensiva, pode-se concluir que: É imprescindível o papel da equipe de enfermagem na prevenção de infecção do trato urinário, pois ela é responsável por todo o manuseio e retirada da sonda vesical de demora, bem como no que se relaciona a adoção de medidas preventivas e campanhas educativas voltadas para o uso correto da sonda vesical, a fim de evitar as ITUs por cateterismo de demora. O estudo evidenciou ainda que através do conhecimento devido dos fatores de risco relacionados à infecção por cateterismo vesical de demora, bem

REAEnf/EJNC | Vol. 6 | e5714 | DOI: https://doi.org/10.25248/REAenf.e5714.2020 Página 5 de 6 
como, as formas de prevenção dessa contaminação, são fundamentais na prestação de uma assistência humanizada adequada, resguardando os pacientes hospitalizados de ações que possam interferir ou exacerbar o processo saúde/doença.

\section{REFERÊNCIAS}

1. ALENCAR PA, et al. Fatores de risco para infecção do trato respiratório e urinário em pacientes de unidade de terapia intensiva. Rev. e-ciência, 8(1): 46-53, 2020.

2. BARBALHO AMDS, et al. Principais antibacterianos utilizados em infecções do trato urinário e seus possíveis riscos durante a gestação: uma revisão. Journal of Medicine and Health Promotion. 2019; 4(4): 1267-1279.

3. BRÍGIDO HPC, et al. Perfil de resistência de agentes de infecção urinária em crianças internadas em um hospital de pediatria em Belém do Pará. Braz. J. Hea. Rev., 2020; 3(4), p. 9808-9818.

4. COMIN D, et al. Prevalência de infecção do trato urinário e perfil de sensibilidade bacteriana aos antimicrobianos prescritos para Gestantes do hospital escola de valença. Revista Saber Digital, 2020; 13(1), p. 70-86.

5. FERRACINI TS, RYMSZA T. Prevalência de infecção urinaria e fatores associados como causadores de trabalho de parto prematuro na fundação hospitalar São Lucas de Cascavel/PR. Revista Thêma et Scientia, 2020; 10(1).

6. HOFFMANN LY, et al. Cistite actínica associada à infecção de trato urinário por Candida glabrata como diagnóstico diferencial de neuralgia do pudendo. Relato de caso. BrJP. São Paulo, 2020; 3(2):182-4.

7. MARKS FO, et al. Infecção do trato urinário: etiologia, perfil de sensibilidade e resistência aos antimicrobianos em hospital pediátrico. Research, Society and Development, 2020; 9(8), e677985807.

8. MARTINS PCS, VAZ AKMG. Infecções prevalentes na unidade de terapia intensiva de um hospital universitário. Enfermagem Brasil 2020;19(3): 238-245.

9. PEREIRA ML, et al. Infecção de trato urinário inferior por Citrobacter sp em cadela com hipoadrenocorticismo. R. bras. Ci. Vet., 2020; 27(2), p. 61-64.

10. PORTELA FM, et al. Infecção urinária como fator de risco para amniorrexe prematura. Revista Cadernos de Medicina, 2019; 2(3).

11. SAKAI AM, et al. Infecção do trato urinário associada ao cateter: fatores associados e mortalidade. Enferm. Foco 2020; 11 (2): 176-181 176.

12. SÉ ACS, et al. Educação em saúde como estratégia de prevenção à infecção do trato urinário associada ao uso de cateter vesical de demora. Research, Society and Development, 2020; 9(9), e453997459.

13. SILVA GF, et al. Prevalence of urinary tract infection on the elderly assisted by a preventive medical care program on Cascavel/PR. FAG Journal of Health, 2020; 3: 352-356.

14. SILVA LR, et al. Infecção do trato urinário em pacientes idosos em atendimento domiciliar: Prevalência, manifestações clínicas e tratamento. REAC/EJSC 2020; 10: e3288.

15. SILVA MR, et al. Infecção de trato urinário associada ao cateterismo vesical de demora na população idosa: classificações de enfermagem. REAEnf/EJNC, 2020; 3: e3540.

16. STELLA AE, OLIVEIRA AF. Padrões de resistência a antibióticos em enterobactérias isoladas de infecções do trato urinário em gestantes. Research, Society and Development, 2020; 9(8): e862986337.

17. VAZ BC, et al. Educação em saúde na prevenção de infecção no trato urinário: relato de experiência. Braz. J. Hea. Rev., Curitiba, 2020; 3(5): p. 13931-13940.

18. SOUZA SM, et al. Infecção do trato urinário (itu) na gestação: deficiências múltiplas $x$ aborto. Revista Saúde e Meio Ambiente - RESMA, 2020; 10(1), p. 19-31.

19. TAVARES JMM, et al. Incidência de infecção urinária em pacientes hospitalizados em uso de cateter vesical de demora. REAS/EJCH, 2020; 12(8): e3497. 